\section{latrogene Sojaallergie}

IgG- bzw. IgG - -Serumanalysen sind für den Nachweis von Nahrungsmittelallergien wegen irrelevanter Ergebnisse abzulehnen. Trotzdem werden die Testkits gerne eingesetzt mit manchmal unangenehmen Folgen. So resultierten die Bemühungen eines Arztes zur Abklärung eines chronischen Hustens in der Feststellung einer „Kuhmilchunverträglichkeit“, Basis für die Diagnose war eine IgG-Analyse. Der Hustenpatient hatte zwar noch nie Probleme nach Kuhmilchkonsum, folgte aber den Ratschlägen zur Ernährungsumstellung. Die gute Compliance wurde mit einer Notfalleinweisung drei Monate später belohnt: Der Mann hatte nach dem Trinken von Sojamilch zunehmend Symptome eines anaphylaktischen Schocks entwickelt. Eine Untersuchung ergab eine Allergie gegen Sojaprotein, Kuhmilchprotein wurde dagegen gut vertragen. Nun ernährt sich der Hustenpatient streng sojaproteinfrei.

Krüger U, Buhl T, Hänßle H, Fuchs T. Fehldiagnose Kuhmilchunverträglichkeit nach IgG-Bestimmung: Schockfragmente auf Sojamilch nach Ernährungsumstellung. Zweiter Gemeinsamer Deutscher Allergiekongress, Lübeck, 26.-29. September 2007

\section{Sogar das Wachstum leidet}

Eine nicht behandelte atopische Dermatitis bei Kindern kann zu schweren Wachstumsverzögerungen führen. Dies zeigt der Fall eines zwölfjährigen Mädchens mit einer nur unzureichend behandelten Neurodermitis und Hausstaubmilbenallergie. Das Mädchen litt während der Nacht unter schwerstem nicht beherrschbaren Juckreiz, was zu jahrelangen ausgeprägten Ein- und Durchschlafstörungen führte. Das Kind war für sein Alter deutlich zu klein, nachgewiesen wurde eine neurosekretorische Dysfunktion mit unzureichender nächtlicher Wachstumshormonsekretion. Nach adäquater Therapie des Ekzems sowie einer Wachstumshormonsubstitution holte die kleine Patientin den Längenrückstand erfreulicherweise fast wieder auf.

End K, Nemat K, Pyper A, Gahr M. Neurosekretorische Dysfunktion und Kleinwuchs als Komplikation einer schwer verlaufenden atopischen Dermatitis bei einem zwölfjährigen Mädchen. Zweiter Gemeinsamer Deutscher Allergiekongress, Lübeck, 26.-29. September 2007 der Hyposensibilisierung auf Symptomatik und Verbrauch topischer Glukokortikoide beim atopischen Ekzem, nach wie vor gibt es aber keine verlässlichen Daten für eine primäre Prävention allergischer Erkrankungen durch eine frühe Immuntherapie der atopischen Dermatitis.

Symposium 1 „Atopisches Ekzem“. Zweiter Gemeinsamer Deutscher Allergiekongress, Lübeck, 26.-29. September 2007

\title{
Je feiner, umso schlimmer?
}

\section{Feinstaub ist in aller Munde - und in den Atemwegen. Der Nachweis der gesundheitlichen Folgen einer hohen Feinstaubexposition hat das Thema zu einem der wichtigsten Umweltprobleme gemacht. Neben Menge und Partikelgröße spielen auch chemisch-physika- lische Eigenschaften des Feinstaubs für die Toxizität eine Rolle.}

\section{F} einstaub ist nicht nur ein durch menschliche Aktivitäten entstandenes Problem. Es gibt ihn auch in natürlicher Form, beispielsweise in den Blue Mountains in Australien, in denen vor allem von Bäumen abgegebene Eukalyptusöle eine hohe Feinstaubbelastung der Luft hervorrufen. Doch für gesundheitliche Folgen in Industrienationen verantwortlich sind meist Feinstäube aus Verbrennungsvorgängen verursacht durch Verkehr, Industrie und Haushalte.

Je nach Partikelgröße lagern sich die Feinstäube in unterschiedlichen Bereichen der Atemwege ab. Im Bereich von Bronchien und Alveolen spielen dabei besonders die Partikel mit einer Größe kleiner $10 \mu \mathrm{m}$ (PM10) und kleiner $5 \mu \mathrm{m}$ (PM5) eine Rolle, diese sind in den letzten Jahren intensiv untersucht worden. Derzeit ist eine neue Direktive in Vorbereitung, in die auch Partikel mit Größen unter 2,5 $\mu \mathrm{m}$ eingehen sollen, berichtete Dr. Thomas A. J. Kuhlbusch vom Institut für Energieund Umwelttechnik in Duisburg. Denn je kleiner die Partikel, desto höher ist die Wahrscheinlichkeit für ein intrazelluläres Eindringen - mit entsprechender toxikologischer Bedeutung.

Die Partikelgröße alleine beschreibt aber noch nicht ausreichend die schädigenden Effekte von Feinstaub. Faktoren wie die Oberfläche der Partikel, ihre Morphologie - z. B. faserartig oder sphärisch -, die Mischung von Substanzen und ihre unterschiedliche Löslichkeit können entscheidend sein für die toxikologische Wirkung. So kann die Struktur der Oberfläche

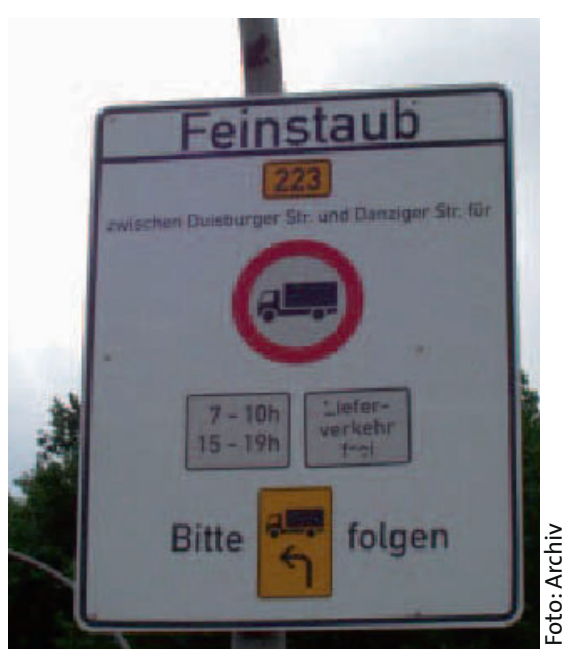

Feinstaub als Umweltrisiko - kann es durch Regulation des Straßenverkehrs reduziert werden?

die Radikalbildung beeinflussen. Oder ein Partikel aus Ammoniumsulfat mit integrierten Russpartikeln setzt diese Partikel nach Auflösung der Ammonium-Schwefel-Verbindung in den Atemwegen frei. Deshalb seien nicht einfach nur die kleinsten Partikel die schlimmsten, erklärte Kuhlbusch. Es komme immer auf das Zusammenspiel der verschiedenen physikalisch-chemischen Partikeleigenschaften unter den Bedingungen der menschlichen Physiologie an.

Symposium 8 ,Bestimmung von inhalativen Expositionen und Belastungen“. Zweiter Gemeinsamer Deutscher Allergiekongress, Lübeck, 26.-29. September 2007 